\title{
Dynamics of Two Sliding Magnetic Surfaces
}

\author{
R.A. Dias, M. Rapini, and B.V. Costa \\ Laboratório de Simulação - Departamento de Física - ICEX - UFMG \\ Caixa Postal 702, 30123-970 Belo Horizonte - MG, Brazil
}

Received on 8 December, 2005

\begin{abstract}
We use Monte Carlo and Molecular Dynamics simulation to study a magnetic tip-sample interaction. Our interest is to understand the mechanism of heat dissipation when the forces involved in the system are magnetic in essence. We consider a magnetic crystalline substrate composed of several layers interacting magnetically with a tip. The set is put thermally in equilibrium at temperature $T$ by using a numerical Monte Carlo technique. By using that configuration we study its dynamical evolution by integrating numerically the equations of motion. Our results suggests that the heat dissipation is closed related to the appearing of vortices in the sample.
\end{abstract}

Keywords: Sliding Nano Magnetic Surfaces; Magnetic Friction; Molecular Dynamic; Monte Carlo

\section{INTRODUCTION}

In magnetic films for which the exchange interaction is less than the separation between layers form quasi 2 dimensional (2d) magnetic planar structures. In general the magnetization of such films is confined to the plane due to shape anisotropy. An exception to that is the appearing of vortices in the system. A vortex being a topological excitation in which the integral of line of the field on a closed path around the excitation core precess by $2 \pi(-2 \pi)$ (See figure 1.). To the purpose of avoiding the high energetic cost of non-aligned moments, the vortices develop three dimensional structures by turning out of the plane the magnetic moment components in the vortex core.[1] For data storage purposes, magnetic vortices are of high interest since its study provides fundamental insight in the mesoscopic magnetic structures of the system[2]. In a data storage device we have always two sliding surfaces in contact: a surface that serves as the storage media and a readwrite head. The quality and the mass of data we can store depend on the capacity of the system to remove heat from the media surface to the neighborhood.
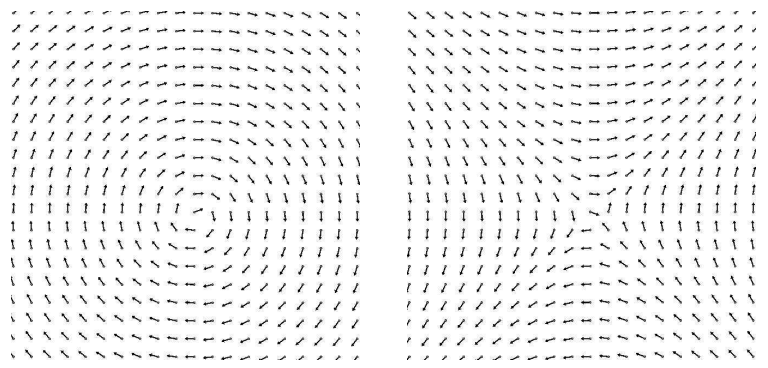

FIG. 1: Schematic view of a vortex (left) and anti-vortex (right) for spins of the same length.

It is our interest in this paper to study the friction between two sliding surfaces. In a macroscopic scale it is known that the force of friction between surfaces satisfies some rules: 1 - The friction is independent of the contacting area between surfaces. 2 - It is proportional to the normal force applied and 3 - The force of kinetic friction is independent of relative speed between surfaces. That behavior being the result of many microscopic interactions between atoms and mole- cules of both surfaces, it is also dependent on the roughness, temperature and energy dissipation mechanisms. Therefore, to understand friction it is necessary to understand its microscopic mechanisms[3].

The availability of refined experimental techniques makes it now possible to investigate the fundamental processes that contribute to the sliding friction on an atomic scale. Issues like how energy dissipates on the substrate, which is the main dissipation channel (electronic or phononic) and how the phononic sliding friction coefficient depends on the corrugation amplitude were addressed, and partially solved, by some groups. [4, 5]. Less known is the effect on friction of a magnetic tip moving relative to a magnetic surface. Applications of sub-micron magnets in spintronics, quantum computing, and data storage demand a huge understand of the sub-micron behavior of magnetic materials. The construction of magnetic devices has to deal with distances of nanometers between the reading head and the storage device. [6-8].

In this paper we use a combined Monte Carlo-Molecular Dynamics (MC-MD) simulation to study the sliding mechanism in a prototype model consisting of a reading head moving close to a magnetic disk surface. A schematic view of our simulation arrangement is shown in figure 2. Our model consists of a magnetic tip (The reading head) which moves close to a magnetic surface (The disk surface.). The tip is simulated
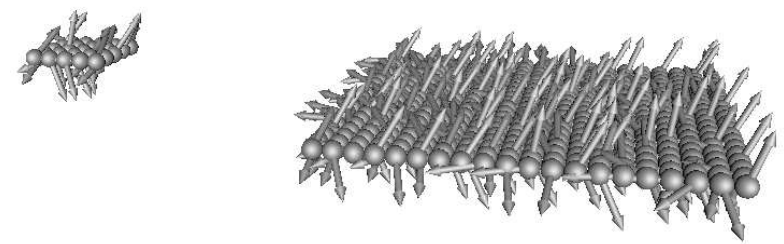

FIG. 2: Schematic view of our simulation arrangement. The tip is simulated as a $5 \times 5$ rigid lattice of particles each carrying a spin $\vec{S}$. The surface is simulated as an arrangement of size $20 \times 20$. The tip is allowed to slid over the surface with initial velocity $v=v_{0} \hat{x}$. The arrows represent the spins directions.

as a cubic arrangement of magnetic dipoles and the surface is represented as a monolayer of magnetic dipoles distributed in a square lattice. We suppose that the dipole interactions are shielded, so that, we do not have to consider them as long 
range interactions. This trick simplifies enormously the calculations putting the cpu time inside reasonable borders.

We fix the sizes of the surface (Fig. 2) and the tip so that only correlations proportional to the length $L_{S}$ of the surface are present. We know that in a real reading head moving close to a magnetic disk surface the correlation length $(\xi)$ is less than the surface's dimension if the system is far from a phase transition, where $\xi$ diverges. However we expect that the phenomenology will not change considerably if we increase the length $L_{s}$ of the surface as long as the tip's length $L_{h}$ is much smaller than $L_{s}$.

The dipole can be represented by classical spin like variables $\vec{S}=S_{x} \hat{x}+S_{y} \hat{y}+S_{z} \hat{z}$. The total energy of this arrangement is a sum of exchange energy, anisotropy energy and the kinetic energy due to the relative movement between the tip and the surface as follows.

$$
H=\sum_{i=1}^{N_{h}} \frac{\vec{p}_{(h-s) i}^{2}}{2 m_{(h) i}}+U_{s p i n}+U_{h-s},
$$

where $U_{\text {spin }}=U_{h}+U_{s}$.

$$
\begin{aligned}
U_{h} & =-\frac{J_{h}}{2} \sum_{<i, j>}\left(S_{h i}^{x} \cdot S_{h j}^{x}+S_{h i}^{y} \cdot S_{h j}^{y}+\lambda_{h} S_{h i}^{z} \cdot S_{h j}^{z}\right) \\
& -D_{h} \sum_{i=1}^{N_{h}}\left(S_{h i}^{z}\right)^{2} \\
U_{s} & =-\frac{J_{s}}{2} \sum_{<i, j>}\left(S_{s i}^{x} \cdot S_{s j}^{x}+S_{s i}^{y} \cdot S_{s j}^{y}+\lambda_{s} S_{s i}^{z} \cdot S_{s j}^{z}\right) \\
& -D_{s} \sum_{i=1}^{N_{s}}\left(S_{s i}^{z}\right)^{2}
\end{aligned}
$$

and

$$
U_{h-s}=-\sum_{i, j} J_{h-s}\left(\left|\vec{r}_{h i}-\vec{r}_{s j}\right|\right)\left(\vec{S}_{h i} \cdot \vec{S}_{s j}\right)
$$

with

$$
J_{h-s}\left(\left|\vec{r}_{h i}-\vec{r}_{s j}\right|\right)=J_{0} \exp \left\{-\alpha\left(\vec{r}_{h i-s j}-r_{0}\right)^{2}\right\}
$$

In equation 1 the first term, $\vec{p}_{(h-s) i}^{2} / 2 m_{(h) i}$, stands for the relative kinetic energy : surface-reading head (s-h). The second term, $U_{\text {spin }}$, account for the magnetic dipoles interactions in the tip, $U_{h}$ and in the surface, $U_{s}$. The last term, $U_{h-s}$, is the interaction energy between the tip and the surface. The symbol $\langle i j\rangle$ means that the sums are to be done over the first neighbors only. For the interaction tip-surface, we suppose that the coupling, $J$, is ferromagnetic. By considering that $J$ is a function of distance, will allow us to study the effects of the relative movement of tip-surface. The exchange anisotropic term $\lambda$, controls the kind of vortex which is more stable in the lattice.

For low values of $\lambda$ the spins close to the center of the vortex are in the plane. For $\lambda>0.7 J$ the configuration that minimizes the vortex energy is for the spins close to the center to develop a large out-of-plane component. Finally, the site anisotropy $D$ controls the out-of-plane magnetization of the model. In the sections below we will discuss behavior of the sliding surface for some values of the parameters. This work is organized as follows. In the section II we introduce general aspects of the numerical method used, in the section III we discuss our results and in section IV we present some conclusions.

\section{SIMULATION BACKGROUND}

In this section we describe the numerical approach we have used to simulate the model. The simulation is done by using a combined Monte Carlo-Molecular Dynamics (MC-MD) procedure. The particles in our simulation move according Newton's law of motion which can be obtained using the hamiltonian 1[9]. The spins evolve according to the equation of motion [10]

$$
\frac{d \vec{S}_{h i, s i}}{d t}=-\vec{S}_{h i, s i} \times \frac{\partial H}{\partial \vec{S}_{h i, s i}} .
$$

As we are mainly interested in the magnetic effects we consider the particles in the tip and in the surface as fixed letting the tip slid over the surface. This generates a set of $3 N+3$ coupled equations of motion which are solved by increasing forward in time the physical state of the system in small time steps of size $\delta t=10^{-3} J^{-1}$. The resulting equations are solved by using Runge-Kutta's method of integration[11].

The surface is arranged as a rigid $20 a \times 20 a$ square lattice with periodic boundary conditions in the $x y$ direction, where $a$ is the lattice spacing. The head is simulated as a rigid $5 a \times 5 a$ square lattice. With no loss of generality the lattice spacing, $a$, will be taken as $a=1$ from now on. In figure 2 we show a schematic view of the arrangement used in our simulation. Initially we put the tip at a large distance from the surface, so that, $J_{h-s}=0$. By using the MC approach we equilibrate the system at a given temperature, $T$. By controlling the energy of the system we found that the system is in equilibrium after $10^{5} \mathrm{MC}$ steps. Once the system is in thermal equilibrium we give an initial velocity, $v=v_{0} \hat{x}$, to the tip. We follow the system's evolution by storing all positions, velocities and spin components at each time step for posterior analysis. From now on the energy is measured in units of $J$, temperature in units of $J / k_{B}$, time in units of $J^{-1}$ and velocity in units of $a / J^{-1}$ where $k_{B}$ is the Boltzmann constant.

\section{RESULTS}

We have simulated the system for several temperatures. For all of them we have fixed $J_{h}=J_{s}=J, J_{0}=2 J, \alpha=1, \lambda_{s}=$ $\lambda_{h}=1$ and the site anisotropy $D_{s}=D_{h}=0.1 J$ to obtain a high out-of-plane component.

With the head far from the surface, we start the time evolution of the system at $t=0$ and with $v_{0}=0$. Up to $t=200$ the head does not interact with the surface. This part of the simulation serves as a guide to the rest of the simulation. Only thermal fluctuations of the vortex density can be seen. At $t=200$ we start moving the head with velocity $v_{0}$. After that, the 

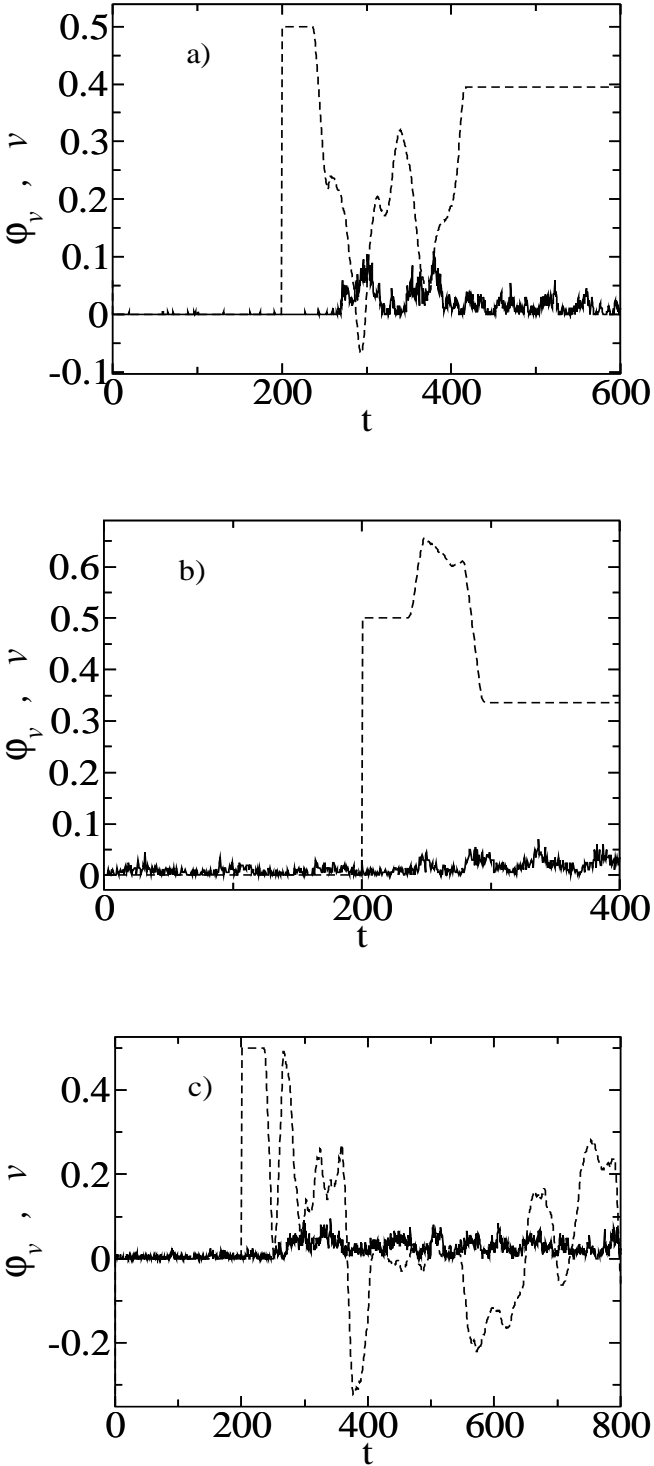

FIG. 3: Instantaneous velocity and vortex density $\left(\varphi_{v}\right)$ as a function of time for $D_{h}=D_{s}=0.1 J$ and $\lambda_{s}=\lambda_{s}=1$. The full line is the vortex density and the dashed line is for the instantaneous velocity. From top to bottom $T=0.1,0.2$. The initial velocity is $v_{0}=0.5$ in all cases.

reading head interact with the surface. We consider that the tip interact with the surface, if there is a non-vanish net force acting on the magnetic head tip and the surface. We will see in the following that depending on the initial velocity, initial configuration and temperature, several things can happen.

In figure 3 we show the velocity of the head and the vortex density $\left(\varphi_{v}\right)$ as a function of time plotted in the same graphic for some interesting situations. The vortex density is multiplied by a constant factor $(f=2)$ as a matter of clarity. The simulations are for $T=0.1$ (Figures 3.a) and $T=0.2$ (Figure 3.b and 3.c). We can observe in the figures that when the head start interacting with the surface the vortex density grows in any situation. That behavior indicates that the formation of vortices can be a mechanism for energy dissipation. In the first plot we observe that the kinetic energy of the tip is trans-
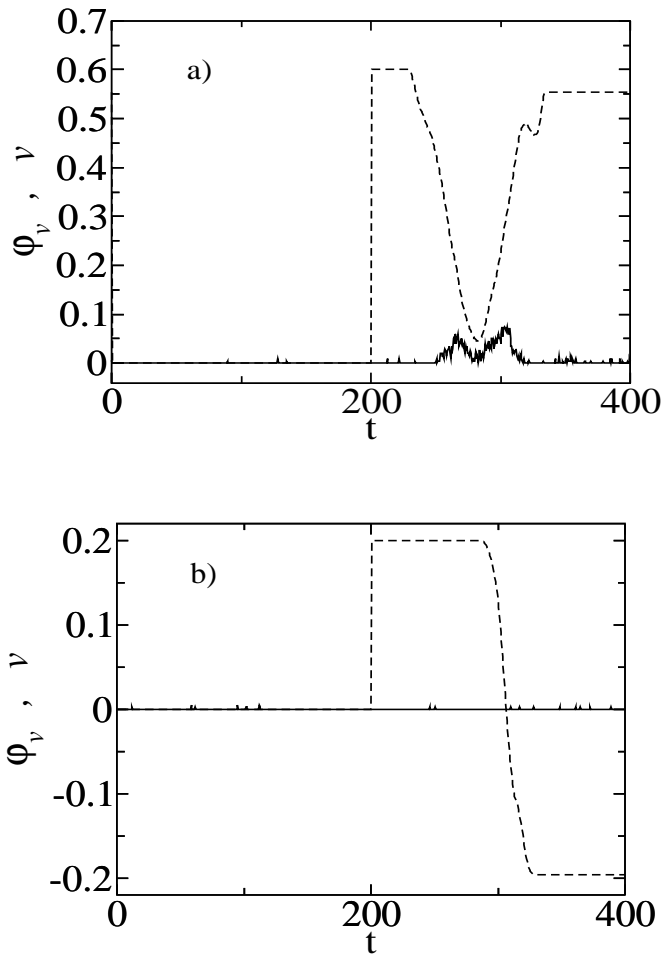

FIG. 4: Instantaneous velocity and vortex density as a function of time for $D_{h}=D_{s}=0.1 J$ and $\lambda_{s}=\lambda_{s}=1$. The full line is the vortex density $\left(\varphi_{v}\right)$ and the dashed line is for the instantaneous velocity. The temperature is $T=0.2$ in both cases. From top to bottom the initial velocity is $v_{0}=0.6,0.2$.

ferred to the surface and the head stops, moves back and forth and escapes from the surface influence. At higher temperatures the situation is a bit more complex. Depending on the initial condition the head can be trapped in the surface region, as seen in figure (3.c), or it passes through it just augmenting the vortex density (3.b). The cost for this increase in the vortex density is a decrease in the kinetic energy.

In figure (4.a) and (4.b) we show the velocity and vortex $\operatorname{density}\left(\varphi_{v}\right)$ as a function of time for different initial velocities $\left(v_{0}=0.6,0.2\right.$ respectively), at a same temperature $T=0.2$. For the higher velocity(4.a), the tip decreases its velocity almost up to stop and become trapped, however its kinetic energy is sufficient to go across the surface region. For a lower temperature and velocity, figure (4.b), the tip interacts almost elastically with the surface.

We note that the increase in the vortex density it is not an instantaneous response to the diminishing of the kinetic energy of the tip. It may be due to an intermediate mechanism: The kinetic energy is used to excite spin waves in the surface. Because there is no mechanism of energy dissipation, part of the energy contained in spin waves is transferred to vortex excitations. 


\section{CONCLUSIONS}

In this work we have simulated the interaction of a magnetic tip with a magnetic surface. Our results show that depending on the relative initial velocity and temperature the tip can interact elastically with the surface, becomes trapped in a small region or pass through the surface region. In the case of a slightly elastic interaction the vortex density does not change. In the cases where the vortex density increases the kinetic energy diminishes. However, the increase in the vortex density it is not an instantaneous response to the diminishing of the kinetic energy of the tip. We suspect that an intermediate mech- anism involving spin waves is present, intermediating the phenomenon.

\section{ACKNOWLEDGMENTS}

We are grateful to L. A. S. Mól for suggestions and comments. Work partially supported by $\mathrm{CNPq}$ and FAPEMIG (Brazilian agencies). Numerical work was done in the LINUX parallel cluster at the Laboratório de Simulação Departamento de Física - UFMG.
[1] J.E.R. Costa and B.V. Costa, Phys. Rev. B 54, (1996)994; J.E.R. Costa, B.V. Costa, and D.P. Landau, Phys. Rev. B 57, (1998)11510; B.V. Costa, J.E.R. Costa, and D.P. Landau, J. Appl. Phys. 81 (1997), 5746.

[2] S.-B Choe, Y. Acremann, A. Scholl, A. Bauer, A. Doran, J. Stöhr, and H. A. Padmore, Science 304,(2004) 402.

[3] E. Meyer, R.M. Overney, K. Dransfeld, and T. Gyalog, Nanoscience - Friction and Rheology on the Nanometer Scale, (World Scientific Publishing, Singapore, 1998).

[4] A. Liebsch, S. Gonçalves, and M. Kiwi, Phys. Rev. B 60,(1999)5034.

[5] E. D. Smith, M. O. Robbins, and M.k Cieplak, Phys. Rev. B $\mathbf{5 4 , ( 1 9 9 6 ) 8 2 5 2 . ~}$
[6] Bo Liu, Jin Liu, and Tow-Chong Chong, J. Mag. Mag. Mater. 287(2005) 339345

[7] A.Y. Suh and A.A. Polycarpou, J. Applied Phys. 97,104328 (2005)

[8] B. Bhushan, J. Magn. Magn. Mater. 155, (1996) 318-322

[9] P.Z. Coura, O.N. Mesquita, and B.V. Costa, Phys. Rev. B 59, (1999) 3408.

[10] D.P. Landau and R.W. Gerling, J. Magn. Magn. Mater. 104-107 (1992)843

[11] D.C. Rapaport, The Art of Molecular Dynamic Simulation (Cambridge University Press, New York, 1997) 